\title{
Time-evolving Weiss fields in the stochastic approach to quantum spins
}

\author{
S. E. Begg $\odot,{ }^{1}$ A. G. Green $\odot,{ }^{2}$ and M. J. Bhaseen $\odot^{1}$ \\ ${ }^{1}$ Department of Physics, King's College London, Strand, London WC2R 2LS, United Kingdom \\ ${ }^{2}$ London Centre for Nanotechnology, University College London, Gordon Street, London WC1H OAH, United Kingdom
}

(Received 10 February 2021; accepted 18 May 2021; published 6 July 2021)

\begin{abstract}
We investigate nonequilibrium quantum spin systems via an exact mapping to stochastic differential equations. This description is invariant under a shift in the mean of the Gaussian noise. We show that one can extend the simulation time for real-time dynamics in one and two dimensions by a judicious choice of this shift. This can be updated dynamically in order to reduce the impact of stochastic fluctuations. We discuss the connection to drift gauges in the gauge- $P$ literature.
\end{abstract}

DOI: 10.1103/PhysRevB.104.024408

\section{INTRODUCTION}

Quantum spin systems play a ubiquitous role in condensedmatter physics, with a myriad of applications ranging from magnetic materials to quantum computers. Out of equilibrium, they exhibit a wealth of phenomena, including anomalous thermalization in low dimensions $[1,2]$ and dynamical quantum phase transitions [3,4]. In one dimension they permit especially strong links between theory and experiment, as exemplified by the recent observation of dynamical quantum phase transitions using a one-dimensional (1D) chain of trapped ions [5]. They have also been instrumental in the development of numerical algorithms, including time-dependent density matrix renormalization group and tensor network approaches [6-8]. These methods have enjoyed widespread applications in one dimension, but they are much harder to apply to nonequilibrium problems in higher dimensions. For state-of-the-art progress in this direction see, for example, [9-12].

Recently, an exact mapping between quantum spin dynamics and classical stochastic differential equations (SDEs) has emerged based upon the Hubbard-Stratonovich decoupling of the exchange interactions [13-19]. This stochastic approach allows for the numerical evaluation of time-dependent quantum observables in addition to analytical insights obtained from the classical stochastic formulas [16-19]. A notable feature is that it treats integrable and nonintegrable problems on a similar footing, including those in higher dimensions. It also offers opportunities for developing links to a diverse body of phase space approaches which have attracted attention in recent years [20-35]. In previous work [18], we showed that the stochastic approach to quantum spins could be significantly improved by a two-patch parameterization of the Bloch sphere, in conjunction with a higher-order numerical integration scheme. We also highlighted the link between the onset of stochastic fluctuations and the non-Hermiticity of the effective stochastic Hamiltonian. In this work, we show that the method for real-time dynamics can be further improved by the use of a dynamical Weiss field to reduce the effects of non-Hermiticity and stochastic fluctuations [18]. In essence, the Weiss field tracks the mean-field dynamics of the quantum spin system, which facilitates more efficient sampling. Similar conclusions have been drawn in imaginary time using saddlepoint techniques [19]. We demonstrate these improvements by presenting results for the quantum Ising model in both one and two dimensions, with up to 121 spins. In the Appendixes, we discuss the link between the SDEs employed here and phase space methods using gauge- $P$ density matrices $[20-22,24,36]$. We show that it is possible to map between the two formalisms using a suitable choice of drift gauge, previously considered for bosonic systems [21,26,36-38]. We conclude with directions for future research.

\section{STOCHASTIC APPROACH}

Recalling the principal steps of Refs. [13,15-18], the stochastic approach can be applied to a generic quadratic spin Hamiltonian

$$
\hat{H}=-\frac{1}{2} \sum_{i j a b} J_{i j}^{a b} \hat{S}_{i}^{a} \hat{S}_{j}^{b}-\sum_{i} h_{i}^{a} \hat{S}_{i}^{a},
$$

where $J_{i j}^{a b}$ is the interaction between spins at lattice sites $i, j$ and $h_{i}^{a}$ is an applied magnetic field. The spin operators $\hat{S}_{i}^{a}$ obey the canonical commutation relations $\left[\hat{S}_{i}^{a}, \hat{S}_{j}^{b}\right]=$ $i \epsilon^{a b c} \delta_{i j} \hat{S}_{j}^{c}$, where $a, b \in\{x, y, z\}$ label the spin components, $\epsilon^{a b c}$ is the antisymmetric symbol, and $\hbar=1$. The interactions in the corresponding time-evolution operator $\hat{U}\left(t_{f}, t_{i}\right)=$ $\mathbb{T} e^{-i \int_{t_{i}}^{t_{f}} \hat{H}(t) d t}$ can be decoupled by performing a HubbardStratonovich transformation over auxiliary fields $\varphi_{j}^{a}$ :

$$
\hat{U}\left(t_{f}, t_{i}\right)=\mathbb{T} \int \mathcal{D} \varphi e^{-S[\varphi]+i \int_{t_{i}}^{t_{f}} d t \sum_{j a} \Phi_{j}^{a} \hat{S}_{j}^{a},}
$$

where $\mathbb{T}$ denotes time ordering. Here, $\mathcal{D} \varphi=\prod_{j a} \mathcal{D} \varphi_{j}^{a}$, and $\Phi_{j}^{a}=\frac{1}{\sqrt{i}} \varphi_{j}^{a}+h_{j}^{a} \in \mathbb{C}$ plays the role of an effective, complex magnetic field. The path integral weight

$$
S[\varphi]=\frac{1}{2} \int_{t_{i}}^{t_{f}} d t \sum_{i j a b} \varphi_{i}^{a}\left(J^{-1}\right)_{i j}^{a b} \varphi_{j}^{b}
$$


is referred to as the noise action [17] since it allows one to interpret the fields $\varphi_{j}^{a}$ as Gaussian distributed random variables. The problem therefore reduces to the dynamics of individual spins coupled to noisy complex fields, where the decoupled spins evolve under the stochastic Hamiltonian, $\hat{H}^{s} \equiv-\sum_{j a} \Phi_{j}^{a} \hat{S}_{j}^{a}$. The spatial and temporal correlations between the spins are encoded in the correlations of the noise fields. By diagonalizing the noise action [15-18] one may introduce new white noise variables $\phi_{j}^{b}$ via $\varphi_{i}^{a} \rightarrow \sum_{j b} O_{i j}^{a b} \phi_{j}^{b}$, where $\boldsymbol{O}^{T} \boldsymbol{J}^{-1} \boldsymbol{O}=\mathbf{1}$; here, we recast $O_{i j}^{a b}$ and $J_{i j}^{a b}$ in terms of matrices $\boldsymbol{O} \equiv O_{(a i)(b j)}$ and $\boldsymbol{J} \equiv \boldsymbol{J}_{(a i)(b j)}$, where (ai) is a twocomponent index.

The stochastic Hamiltonian gives rise to a stochastic evolution operator $\hat{U}^{s}(t)=\mathbb{T} e^{-i \int_{0}^{t} \hat{H}^{s}\left(t^{\prime}\right) d t^{\prime}} \equiv \prod_{j} \hat{U}_{j}^{s}(t)$, which factorizes into on-site contributions. Using the Lie algebraic structure of $\hat{H}^{s}$, we may parametrize $\hat{U}_{j}^{s}(t)$ via a so-called disentanglement transformation: $\hat{U}_{j}^{s}(t)=e^{\xi_{j}^{+}(t) \hat{S}_{j}^{+}} e^{\xi_{j}^{z}(t) \hat{S}_{j}^{z}} e^{\xi_{j}^{-}(t) \hat{S}_{j}^{-}}$ [15]. The $\xi$ variables evolve according to SDEs:

$$
\begin{aligned}
-i \dot{\xi}_{j}^{+} & =\Phi_{j}^{+}+\Phi_{j}^{z} \xi_{j}^{+}-\Phi_{j}^{-} \xi_{j}^{+^{2}}, \\
-i \dot{\xi}_{j}^{z} & =\Phi_{j}^{z}-2 \Phi_{j}^{-} \xi_{j}^{+}, \\
-i \dot{\xi}_{j}^{-} & =\Phi_{j}^{-} e^{\xi_{j}^{z}},
\end{aligned}
$$

where $\Phi_{j}^{ \pm}=\frac{1}{2}\left(\Phi_{j}^{x} \mp i \Phi_{j}^{y}\right)$. The latter can be written in terms of the white noise variables as $\Phi_{j}^{a}=\frac{1}{\sqrt{i}} \sum_{j b} O_{i j}^{a b} \phi_{j}^{b}+h_{j}^{a}$, where [15-18]

$$
\left\langle\phi_{i}^{a}(t) \phi_{j}^{b}\left(t^{\prime}\right)\right\rangle=\delta_{a b} \delta_{i j} \delta\left(t-t^{\prime}\right), \quad\left\langle\phi_{i}^{a}(t)\right\rangle=0 .
$$

To calculate quantum observables, $\langle\hat{\mathcal{O}}(t)\rangle=$ $\left\langle\psi(0)\left|\hat{U}^{\dagger} \hat{\mathcal{O}} \hat{U}\right| \psi(0)\right\rangle$, both the forwards and backwards time-evolution operators must be independently decoupled [16]. Observables thereby reduce to averages of functions of the associated decoupling fields, $\xi$ and $\widetilde{\xi}$ [16]. To solve the SDEs (4a) and (4b), we use the Heun predictor-corrector integration scheme in the Stratonovich formalism [39,40], with a time step $d t=0.01$, unless stated otherwise. We also remove coordinate singularities via the two-patch approach given in [18].

\section{EFFECTIVE WEISS FIELD}

A key feature of the representation (2) is that it is invariant under shifts of the Hubbard-Stratonovich fields $\varphi(t) \rightarrow \varphi(t)+\Delta \varphi(t)$ since the fields correspond to dummy integration variables in the path integral. This leaves the time-evolution operator unchanged, which was recently used to develop an importance sampling approach in imaginary time $[19,41]$. In this work, we show that a judicious choice of $\Delta \varphi(t)$ can significantly improve numerical simulations of real-time dynamics over a broad range of parameters. To gain some intuition for this, we note that under this transformation, the effective magnetic field transforms as $\Phi_{i}^{a} \rightarrow \frac{1}{\sqrt{i}} \sum_{j b} O_{i j}^{a b}\left(\phi_{j}^{b}+\Delta \phi_{j}^{b}\right)+h_{i}^{a}$. Denoting $\Delta \phi_{j}^{b}=\sqrt{i} \sum_{k c} m_{k}^{c} O_{k j}^{c b}$, this can be rewritten as $\Phi_{i}^{a}=$ $\frac{1}{\sqrt{ } i} \sum_{j b} O_{i j}^{a b} \phi_{j}^{b}+h_{i}^{a}+\sum_{j b} J_{i j}^{a b} m_{j}^{b}$. At this stage the parameter $m_{j}^{b}$ is completely arbitrary. However, as we will expand upon in Secs. IV and V and the contribution $\sum_{j b} J_{i j}^{a b} m_{j}^{b}$ can be

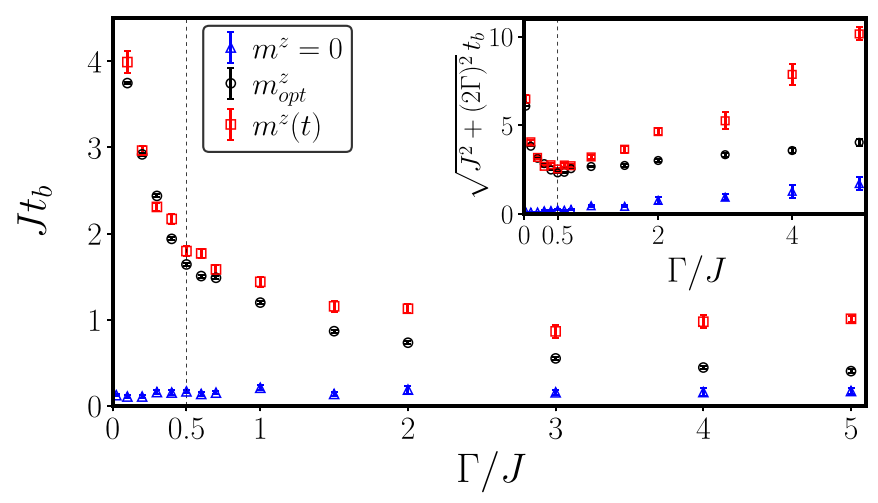

FIG. 1. Comparison of the breakdown time $t_{b}$ for simulations of the $1 \mathrm{D}$ quantum Ising model with 10 spins, following a quantum quench from the fully polarized initial state $|\Downarrow\rangle$ to different values of $\Gamma / J$. The results are obtained by numerical solution of the SDEs without a Weiss field $\left(m^{z}=0\right)$, in the presence of an optimal static Weiss field $\left(m_{\mathrm{opt}}^{z}\right)$, and with a time-evolving Weiss field $m^{z}(t)$. The use of a Weiss field leads to longer simulation times. Inset: rescaling $t_{b}$ by $\sqrt{J^{2}+(2 \Gamma)^{2}}$, which is proportional to the Hilbert-Schmidt norm of the Ising Hamiltonian $\left\|\hat{H}_{I}\right\|$, facilitates the comparison of $t_{b}$ for different values of $\Gamma / J$. With this rescaling, the smallest breakdown time for both static and time-evolving Weiss fields occurs at the critical point $\Gamma=J / 2$.

interpreted as an effective Weiss field due to the neighboring spins. For example, in the special case of isotropic nearestneighbor interactions, this reduces to $Z J^{a b} m^{b}$, where $m^{b}=m_{j}^{b}$ and $Z$ is the coordination number. This mirrors the mean-field contribution of neighboring spins to the local Weiss field, where $m_{j}^{b}$ is the component of the magnetization in the direction specified by $b$. More generally, we may choose the parameter $m_{j}^{b}(t)$ to be time dependent, in accordance with the dynamics of the neighboring spins. The shift of the fields $\varphi$ also induces a transformation of the probability measure via the noise action (3) [19,41]:

$$
S[\phi] \rightarrow S[\phi, m]=S[\phi]+\Delta S[\phi, m],
$$

where

$$
\Delta S=\frac{1}{2} \int_{t_{i}}^{t_{f}} d t\left(2 \sqrt{i} \sum_{i j a b} m_{i}^{a} O_{i j}^{a b} \phi_{j}^{b}+i \sum_{i j a b} J_{i j}^{a b} m_{i}^{a} m_{j}^{b}\right)
$$

and $S[\phi]=\frac{1}{2} \int_{t_{f}}^{t_{f}} d t \sum_{i a}\left(\phi_{i}^{a}\right)^{2}$ is the diagonal form of the noise action. This reweights the stochastic trajectories by terms involving the dynamical Weiss field $m_{i}^{a}(t)$.

In Fig. 1 we highlight the improvements obtained by the use of a Weiss field. Figure 1 shows the breakdown time of numerical simulations $t_{b}$ following a quantum quench in the 1D quantum Ising model

$$
\hat{H}_{I}=-\frac{1}{2} \sum_{\langle i j\rangle} J_{i j} \hat{S}_{i}^{z} \hat{S}_{j}^{z}-\Gamma \sum_{j=1}^{N} \hat{S}_{j}^{x},
$$

with $N=10$ spins and nearest-neighbor interactions $J_{i j}=J$, from the fully polarized state $|\Downarrow\rangle \equiv \prod_{j}|\downarrow\rangle_{j}$ to different values 
of $\Gamma / J$. The relevant SDEs are

$$
\begin{aligned}
-i \dot{\xi}_{j}^{+} & =\frac{\Gamma}{2}+\left(\frac{1}{\sqrt{i}} \sum_{k} O_{j k}^{z z} \phi_{k}^{z}+\sum_{k} J_{j k} m_{k}^{z}\right) \xi_{j}^{+}-\frac{\Gamma}{2} \xi_{j}^{+^{2}}, \\
-i \dot{\xi}_{j}^{z} & =\frac{1}{\sqrt{i}} \sum_{k} O_{j k}^{z z} \phi_{k}^{z}+\sum_{k} J_{j k} m_{k}^{z}-\Gamma \xi_{j}^{+}, \\
-i \dot{\xi}_{j}^{-} & =\frac{\Gamma}{2} e^{\xi_{j}^{z}} .
\end{aligned}
$$

In practice, the variable $\xi_{j}^{-}$can be neglected since it drops out of observables involving the initial spin-down state at site $j$ [18]. The data in Fig. 1 correspond to (i) the SDEs without a Weiss field $\left(m_{j}^{z}=0\right)$; (ii) an optimal choice of spatially uniform static Weiss field, as discussed in Sec. IV; and (iii) a spatially uniform time-evolving Weiss field $m^{z}(t)$, which is determined self-consistently in Sec. V. The key point is that the use of a Weiss field leads to longer breakdown times over a broad range of parameters. In the remainder of this work, we will consider each of these cases in turn. In Sec. IV we consider the case where $m_{j}^{b}$ is spatially homogeneous and static and investigate its impact upon the dynamics of quantum expectation values. In Sec. V we consider timedependent extensions via a self-consistent choice of $m_{j}^{b}(t)$. In the Appendixes, we demonstrate that the generalized SDEs, including a Weiss field, can be obtained within the gauge- $P$ approach for a particular choice of drift gauge.

\section{STATIC WEISS FIELD}

In this section we explore the improvements in numerical simulations obtained through the use of a static Weiss field. We consider quantum quenches in the 1D quantum Ising model (8), with periodic boundary conditions and $J=1$. We start in the fully polarized initial state $|\Downarrow\rangle=\prod_{i=1}^{N}|\downarrow\rangle$ and quench to different values of $\Gamma / J$. The expectation value of the spin operator $\hat{\mathbf{S}}_{j}$ has an intuitive representation in the stochastic approach [18]:

$$
\left\langle\hat{\mathbf{S}}_{j}(t)\right\rangle=\left\langle\mathcal{W} \prod_{i}\left|\psi_{i}^{s}(t)\right|^{2} \mathbf{n}_{j}(t)\right\rangle_{\phi, \tilde{\phi}},
$$

where $\langle\cdots\rangle_{\phi, \tilde{\phi}}$ denotes averaging over the Gaussian white noise variables. The weight $\mathcal{W}=e^{-\Delta S[\phi, m]-\Delta S^{*}[\tilde{\phi}, m]}$ is discretized in time, and it weights the stochastic trajectories via the Weiss field. This mirrors the reweighting of trajectories performed in imaginary time [19]. The vector $\mathbf{n}_{j}(t)$ corresponds to the position of a spin on the Bloch sphere, expressed in terms of projective coordinates [18]:

$$
\mathbf{n}_{j}(t)=\frac{1}{2}\left(\frac{2 \operatorname{Re}\left[\xi_{j}^{+}(t)\right]}{1+\left|\xi_{j}^{+}(t)\right|^{2}}, \frac{-2 \operatorname{Im}\left[\xi_{j}^{+}(t)\right]}{1+\left|\xi_{j}^{+}(t)\right|^{2}}, \frac{-1+\left|\xi_{j}^{+}(t)\right|^{2}}{1+\left|\xi_{j}^{+}(t)\right|^{2}}\right) .
$$

The factor of $\left|\psi_{i}^{s}(t)\right|^{2}$ corresponds to the norm of the stochastic state $\left|\psi_{i}^{s}(t)\right\rangle=\hat{U}_{i}^{s}(t)|\psi(0)\rangle$ and is given by

$$
\left|\psi_{i}^{s}(t)\right|^{2}=e^{-\operatorname{Re}\left[\xi_{i}^{z}(t)\right]}\left[1+\left|\xi_{i}^{+}(t)\right|^{2}\right] .
$$

In writing (11) and (12), it is implicit that the conjugate variable $\xi_{j}^{a *}$ is independent of $\xi_{j}^{a}$; we denote this via the
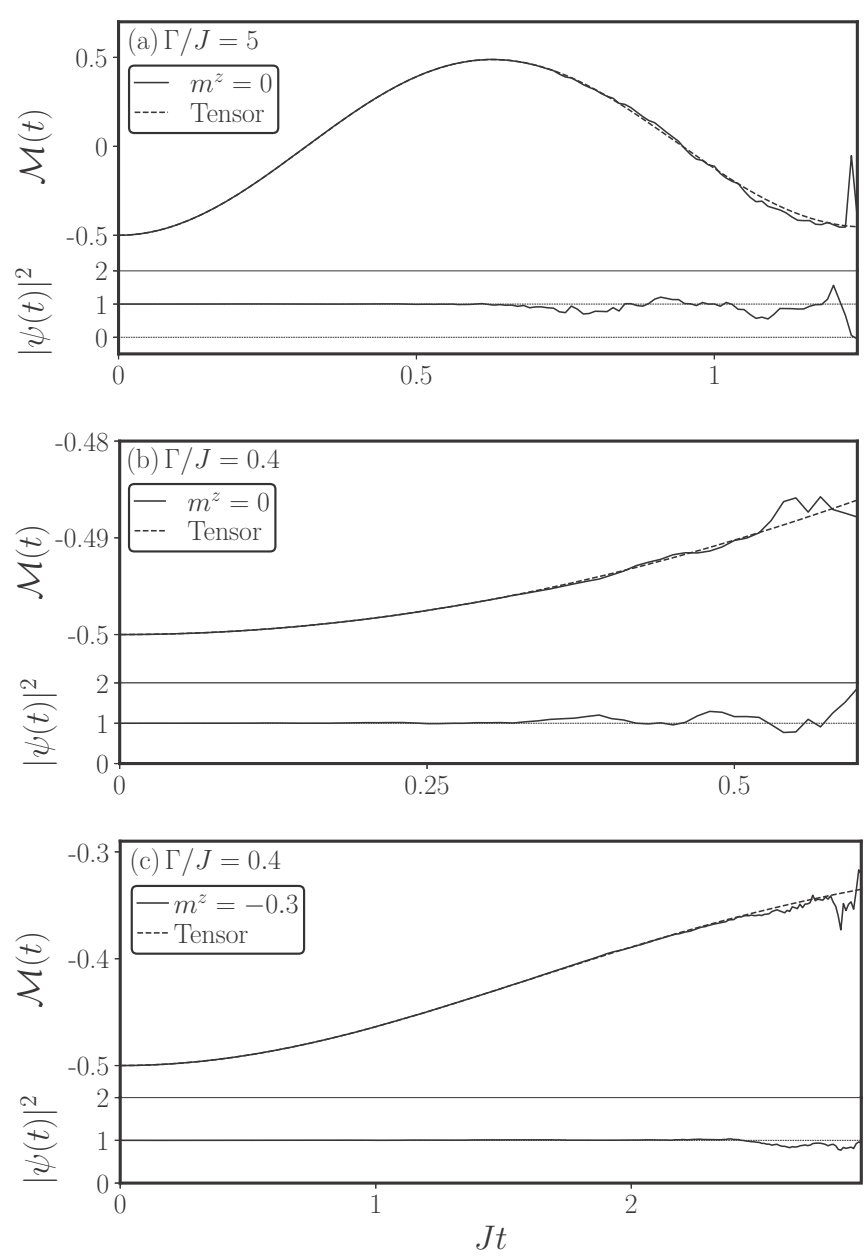

FIG. 2. Time evolution of the magnetization $\mathcal{M}(t)$ following a quantum quench from the fully polarized initial state $|\Downarrow\rangle$ in the 1D quantum Ising model with 25 sites. (a) Quench to $\Gamma / J=5$ using the SDEs in the absence of a Weiss field with $m^{z}=0$ (solid line) and using tensor network matrix product operator methods (dashed line). (b) Quench to $\Gamma / J=0.4$ with $m^{z}=0$ (solid line) and using tensor networks (dashed line). The accessible timescale for the stochastic approach is reduced in comparison to (a). This can be extended by using a well-chosen static Weiss field. (c) Quench to $\Gamma / J=0.4$ with $m^{z}=-0.3$ (solid line) showing improved simulation times. In all the cases we average over $\mathcal{N}=10^{6}$ trajectories. The norm of the quantum state is also shown to indicate the reliability of the simulations, and the eventual breakdown time.

replacement $\xi_{j}^{a *} \rightarrow \tilde{\xi}_{j}^{a *}$. Although (10) is formally exact, the norm of the quantum state is not preserved in numerical simulations with a finite number $\mathcal{N}$ of stochastic samples [18]. As such, we further rescale by the quantum state norm [18]

$$
|\psi(t)|^{2}=\left\langle\mathcal{W} \prod_{i}\left|\psi_{i}^{s}(t)\right|^{2}\right\rangle_{\phi, \tilde{\phi}} .
$$

In Fig. 2(a) we show the time dependence of the magnetization, $\mathcal{M}(t)=\frac{1}{N} \sum_{j=1}^{N}\left\langle\hat{S}_{j}^{z}\right\rangle$, with $N=25$, following a quantum quench from the initial state $|\Downarrow\rangle$ to the paramagnetic phase with $\Gamma=5 J$. The results are obtained in the absence of a Weiss field $\left(m_{j}^{z}=0\right)$ and are in excellent agreement with those obtained via the tensor network matrix product operator 

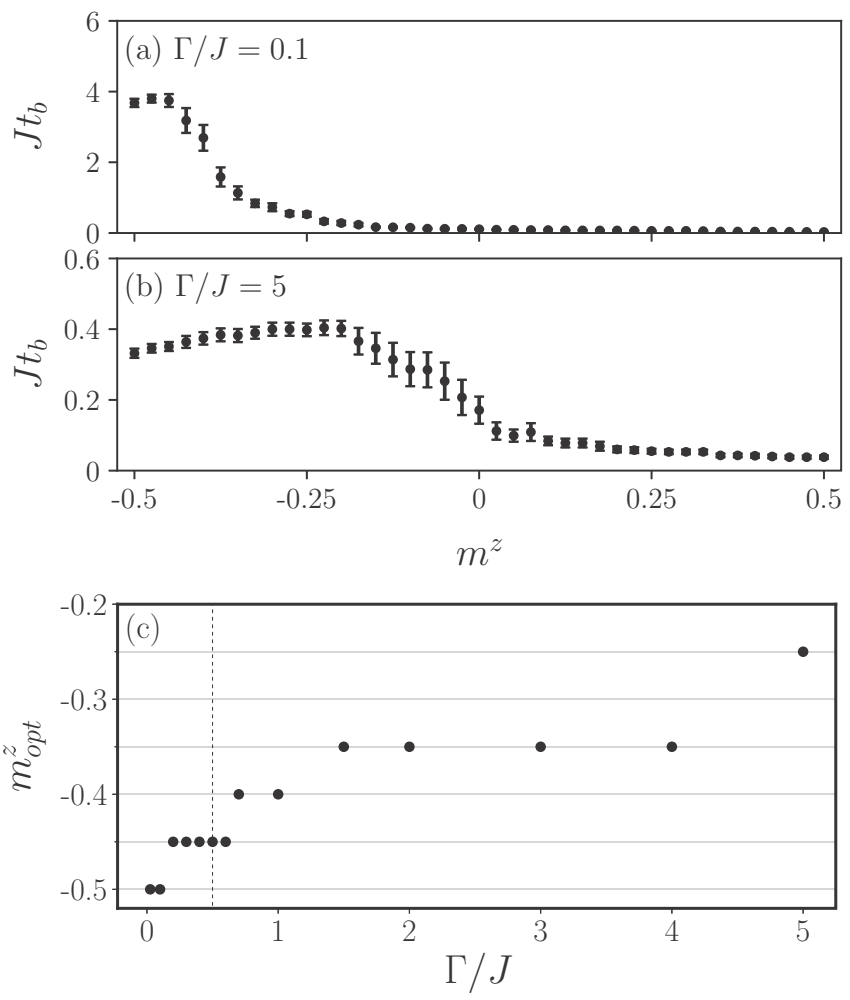

FIG. 3. Breakdown time $t_{b}$ versus the static Weiss field $m^{z}$ for quenches in the 1D quantum Ising model with 10 spins from the fully polarized state $|\Downarrow\rangle$ to (a) $\Gamma / J=0.1$ and (b) $\Gamma / J=5$. The optimal Weiss field, corresponding to the longest simulation time, is given by $m_{\mathrm{opt}}^{z} \approx-0.45$ in (a) and $m_{\mathrm{opt}}^{z} \approx-0.2$ in (b). (c) Variation of $m_{\mathrm{opt}}^{z}$ as a function of the postquench value of $\Gamma / J$. The optimal value is selected from the range $-0.5 \leqslant m_{\mathrm{opt}}^{z} \leqslant 0$, which is discretized in steps of 0.05 . The breakdown times are obtained as the average of the breakdown time from 10 batches of $\mathcal{N}=10,000$ runs. The error bars in (a) and (b) correspond to the standard error of these batches.

technique (MPO) $W^{I}$ [42] for the time-interval displayed. For comparison, we also show the norm of the time-evolving quantum state as calculated via (13). It is readily seen that departures from coincidence occur when the norm deviates from unity [18]. Throughout this work, we define the breakdown time $t_{b}$ of our numerical simulations as the time at which this deviation reaches 1\%. In Fig. 2(b), we show results for a quench to $\Gamma=0.4 \mathrm{~J}$, within the ferromagnetic phase. The results are obtained in the absence of a Weiss field $\left(m_{j}^{z}=0\right)$ and break down at an earlier time than those in Fig. 2(a). For comparison, in Fig. 2(c) we show results for the same quench as in Fig. 2(b) but in the presence of a static Weiss field $m_{j}^{z}=-0.3$; as we will discuss below, this turns out to be a near-optimal choice of the static Weiss field for this particular quench. Since the model (8) contains only $z$ interactions, we consider Weiss fields in only the $z$ direction. It is evident that the simulation time is extended beyond that in Figs. 2(a) and 2(b).

In order to gain some insight into the variation of the breakdown time $t_{b}$ with $m^{z}=m_{j}^{z}$, we consider quenches to different points in the phase diagram as a function of $m^{z}$. To aid the comparison, we fix the number of stochastic samples to $\mathcal{N}=10000$. In Fig. 3(a) we plot the dimensionless breakdown time $J t_{b}$ versus $m^{z}$ for a quench to $\Gamma=0.1 J$ within the ferromagnetic phase. It can be seen that the best choices for the static Weiss field lie in the range $-0.5 \lesssim m^{z} \lesssim-0.35$. In Fig. 3(b) we do the same analysis for $\Gamma=5 J$. It can be seen that this larger value of $\Gamma$ reduces the magnitude of the optimal choice for $m^{z}$. In Fig. 3(c) we show the variation of the optimal Weiss field $m_{\mathrm{opt}}^{z}$ for quenches to different points in the phase diagram. It can be seen that the optimal choice of $m^{z}$ interpolates between $m^{z}=-1 / 2$ and $m^{z}=0$ as one passes from the ferromagnetic region $(\Gamma<J / 2)$ to the paramagnetic region $(\Gamma>J / 2)$.

In Fig. 1 we show the breakdown time corresponding to the optimal static Weiss field. It can be seen that the use of a Weiss field leads to a significant improvement in the simulation time throughout the phase diagram. The inset shows the same data rescaled by $\sqrt{J^{2}+(2 \Gamma)^{2}}$, which is proportional to the Hilbert-Schmidt norm of the Ising Hamiltonian $\left\|\hat{H}_{I}\right\|_{2}=\sqrt{\operatorname{Tr}\left(\hat{H}_{I}^{2}\right)}$ [43]. This facilitates the comparison of the timescales for different quantum quenches. It can be seen that the shortest rescaled simulation times occur for quenches close to the quantum critical point at $\Gamma=J / 2$, as one would naïvely expect due to enhanced fluctuations.

\section{TIME-EVOLVING WEISS FIELD}

In this section we examine the possibility of choosing the value of $m_{j}^{z}$ as a function of time. A natural choice is evident if we write the stochastic Hamiltonian for the quantum Ising model in the form

$$
\begin{aligned}
\hat{H}^{s}(t)= & -\sum_{i} \Gamma \hat{S}_{i}^{x}-\sum_{i j} J_{i j} m_{j}^{z}(t) \hat{S}_{i}^{z} \\
& -\frac{1}{\sqrt{i}} \sum_{i} \varphi_{i}^{z}\left[\hat{S}_{i}^{z}-m_{i}^{z}(t) \hat{\mathbb{I}}\right],
\end{aligned}
$$

where $\hat{\mathbb{I}}$ is the identity operator and $\varphi_{i}^{z}$ is the original decoupling field with probability measure (3). The additional terms that would arise via (7) have been absorbed into $\hat{H}^{s}(t)$; the $\mathcal{O}\left(\mathrm{m}^{2}\right)$ terms can be neglected since they result in a deterministic phase for $\left|\psi^{s}(t)\right\rangle$ which is identical for all trajectories. Choosing $m_{j}^{z}(t)$ to be the instantaneous average of $\left\langle\hat{S}_{j}^{z}\right\rangle$ allows one to reduce the effects of non-Hermiticity arising from (14):

$$
m_{j}^{z}(t)=\left\langle\frac{\left\langle\psi^{s}(t)\left|\hat{S}_{j}^{z}\right| \psi^{s}(t)\right\rangle}{\left|\psi^{s}(t)\right|^{2}}\right\rangle_{\phi},
$$

where the average is over the noise variables associated with the forwards time evolution; the Weiss field for the backwards evolution takes the same value. Enforcing the Bloch-sphere normalization explicitly in (15) results in contributions to the average that are comparable in size. The result therefore converges with far fewer samples than are needed for quantum observables such as (10). The choice (15) also generates the physically transparent mean-field term $\sum_{i j} J_{i j} m_{i}^{z} \hat{S}_{j}^{z}$ in the stochastic Hamiltonian (14). This is analogous to the optimal shift for imaginary-time evolution, corresponding to a meanfield saddle point [19]. Since $\left|\psi^{s}(t)\right\rangle$ is itself a function of $m_{j}^{z}(t)$, the Weiss field should be determined iteratively. To do this, we first set $m_{j}^{z}(t)=0$ and simulate trajectories to yield (15). This is then used as $m_{j}^{z}(t)$ for the next simulation. 


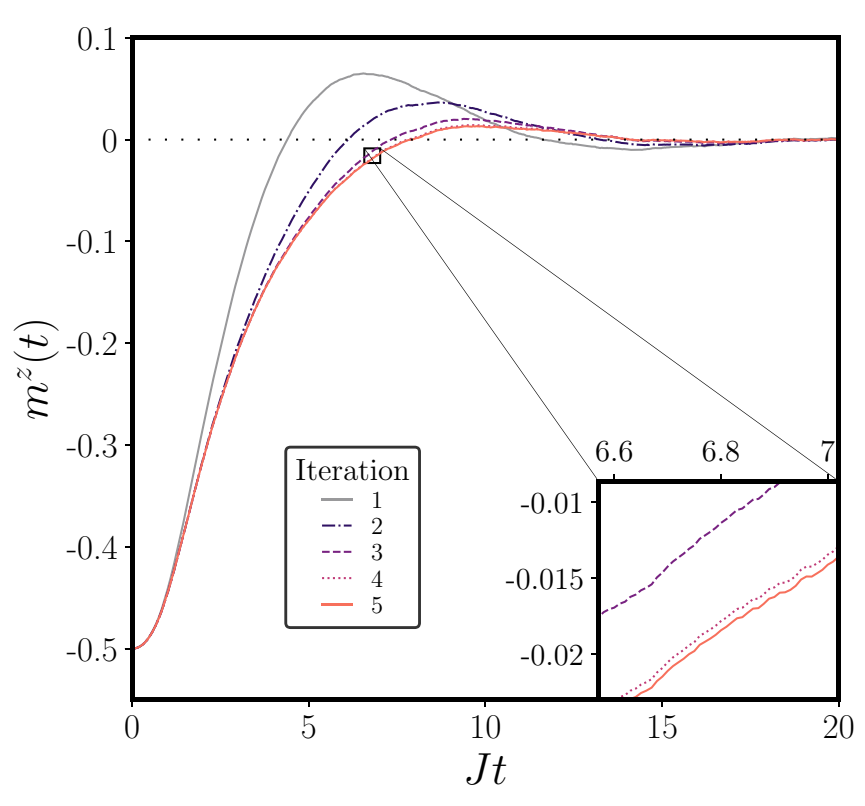

FIG. 4. Determination of the time-evolving Weiss field $m^{z}(t)$ following a quantum quench in the $1 \mathrm{D}$ quantum Ising model with 10 sites from the fully polarized state $|\Downarrow\rangle$ to $\Gamma / J=0.4$. The results correspond to five iterations of the procedure discussed in the text, where each iteration corresponds to $\mathcal{N}=10000$ stochastic trajectories. As shown in the inset, the results converge to a fixed time-dependent profile for $m^{z}(t)$.

We proceed in this iterative fashion until $m_{j}^{z}(t)$ converges to a fixed time evolution. For translationally invariant states, we may consider a single Weiss field $m^{z}(t)=\frac{1}{N} \sum_{j=1}^{N} m_{j}^{z}(t)$ applied to all the sites. As we discuss in Appendix E, one can estimate this field from a small subsystem that captures the local interactions.

In Fig. 4 we plot $m^{z}(t)$ as a function of time for simulations of the $1 \mathrm{D}$ quantum Ising model with $N=10$ spins. We consider a quantum quench from the fully polarized state $|\Downarrow\rangle$ to $\Gamma=0.4 J$, showing the results from each iteration. After four iterations of $\mathcal{N}=10000$ samples the data converge to a fixed-point value of $m^{z}(t)$, to a high level of accuracy. As shown in Fig. 1, the time-evolving Weiss field performs at least as well as the optimal static choice. For small $\Gamma / J$, a key advantage of the time-dependent procedure is that one does not have to survey different static Weiss fields. For $\Gamma \gg J$ the performance of $m^{z}(t)$ is superior to $m_{\mathrm{opt}}^{z}$, as it self-consistently tracks the mean-field dynamics. In comparison, the optimal static Weiss field, $m_{\mathrm{opt}}^{z}=0$, captures only the time average of the time-evolving mean field.

\section{IMPLEMENTATION}

Having established a protocol for determining the timeevolving Weiss field $m^{z}(t)$, we now explore its effectiveness in numerical simulations. We focus on moderately large system sizes in both one and two dimensions. Throughout this section, the Weiss field is determined by four iterations of the self-consistent approach with a relatively small number of $\mathcal{N}=1000$ samples. In Fig. 5(a) we show results for the transverse magnetization, $\mathcal{M}^{x}(t)=\frac{1}{N} \sum_{i}\left\langle\hat{S}_{i}^{x}\right\rangle$, following a
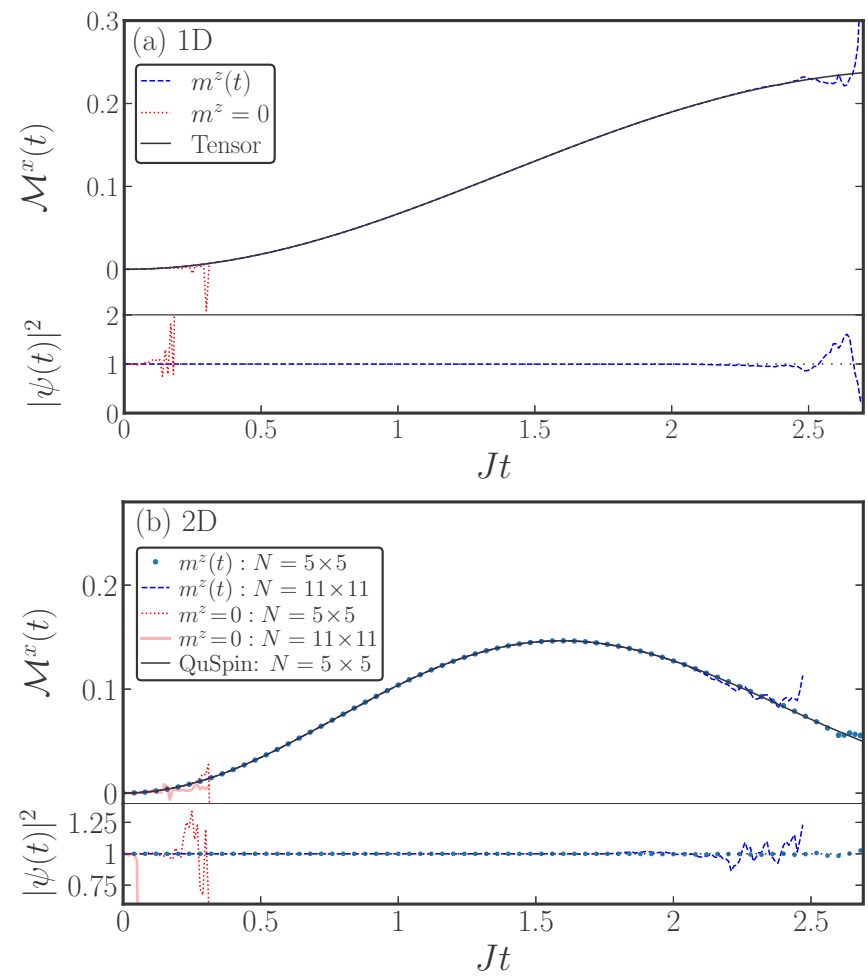

FIG. 5. Dynamics of the transverse magnetization $\mathcal{M}^{x}(t)$ following a quantum quench in the (a) $1 \mathrm{D}$ and (b) $2 \mathrm{D}$ quantum Ising models, from the fully polarized initial state $|\Downarrow\rangle$ to $\Gamma / J=0.3$. (a) One-dimensional results for a 101-site system obtained from the SDEs using a time-dependent Weiss field $m^{z}(t)$ and $\mathcal{N}=5 \times 10^{5}$ trajectories (dashed line). The results are in agreement with those obtained via tensor networks (solid line) until the breakdown time. It can be seen that the results for $m^{z}=0$ (dotted line) break down earlier. (b) Two-dimensional results for a $5 \times 5$ lattice with $\mathcal{N}=10^{6}$ (dots) and an $11 \times 11$ lattice with $\mathcal{N}=5 \times 10^{5}$ (dashed line). The former are in agreement with the results obtained via QuSpin's ODE solver [44] (solid line). In the absence of a comparison to other techniques, the $11 \times 11$ results are seen to be in good agreement with the $5 \times 5$ results until the breakdown time; beyond this, strong fluctuations occur in the $11 \times 11$ case. Once again, the $m^{z}=0$ results (red dotted and light solid lines) break down earlier. In all cases, the results are plotted until fluctuations cause departures from the true dynamics. In each panel, the time-dependent Weiss field $m^{z}(t)$ is obtained by four iterations of the procedure discussed in the main text using $\mathcal{N}=10^{3}$ stochastic samples.

quantum quench from $|\Downarrow\rangle$ to $\Gamma=0.3 J$, in the $1 \mathrm{D}$ quantum Ising model with $N=101$ spins. The results are in very good agreement with tensor network methods until $J t_{b}=2.11$; this is a significant improvement over the $m^{z}=0$ case where $J t_{b}=0.05$. In Fig. 5(b) we show results for $\mathcal{M}^{x}(t)$ following a quantum quench in the $2 \mathrm{D}$ quantum Ising model with $N=$ $5 \times 5$ and $N=11 \times 11$ sites. In the former case, the results are in excellent agreement with those obtained via QuSpin's ordinary differential equation (ODE) solver [44] until $J t_{b}=2.56$; this exceeds the $m^{z}=0$ case, which has a breakdown time of $J t_{b}=0.09$. In the absence of another method with which to compare, the results for the $N=11 \times 11$ case are compared to those obtained for smaller system sizes. The data track each 
other until the breakdown time $t_{b}$, suggesting that the results for the larger system size are reliable. There is a similarly large improvement over the $m^{z}=0$ case.

\section{CONCLUSIONS}

In this work, we have introduced time-evolving Weiss fields into the stochastic approach for real-time quantum spin dynamics. We have shown that they can significantly extend the timescales for numerical simulations in both one and two dimensions. In the Appendix, we have further demonstrated that these Weiss fields can be obtained via the use of drift gauges in the gauge- $P$ phase space formulation. It would be interesting to explore this connection in future work.

Note added in proof. Recently, we became aware of forthcoming work [46] which extends [19] to real time.

\section{ACKNOWLEDGMENTS}

We acknowledge helpful discussions with F. Barratt and S. De Nicola. M.J.B. acknowledges stimulating conversations with D. O'Dell and S. Wüster at the ICTS (Bengaluru) program on Non-Hermitian Physics PHHQP XVIII. S.E.B. is supported by the EPSRC CDT in Cross-Disciplinary Approaches to Non-Equilibrium Systems (CANES) via Grant No. EP/L015854/1. We are grateful to the UK Materials and Molecular Modelling Hub for computational resources, which is partially funded by EPSRC (Grant No. EP/P020194/1). The MPO calculations were performed using the ITensor Library [45]. A.G.G. acknowledges EPSRC Grant No. EP/P013449/1. M.J.B. acknowledges the support of the London Mathematical Laboratory.

\section{APPENDIX A: FOKKER-PLANCK DESCRIPTION}

As highlighted in the main text, we can make contact between the SDEs employed here and the gauge- $P$ approach [20,22-24], through the use of drift gauges [21]. To see this, we first consider the Fokker-Planck description of the stochastic approach to quantum spin systems [13]. This will enable us to develop connections to a broad class of "phase space" methods, which describe quantum systems via mappings to classical coordinates.

As usual, one may switch between a Langevin-type description of a stochastic process and a Fokker-Planck description by introducing a probability distribution $P(\xi)$ for the stochastic variables $\xi$. For a quantum spin system, this can be introduced by means of the density matrix $\hat{\rho}=\left\langle\hat{\rho}^{s}\right\rangle_{\phi, \tilde{\phi}}$, where $\hat{\rho}^{s}$ is the stochastic density matrix $\hat{\rho}^{s}=\left|\psi^{s}(\phi, t)\right\rangle\left\langle\psi^{s}(\tilde{\phi}, t)\right|$. More explicitly,

$$
\hat{\rho}^{s}=\hat{U}^{s}(\phi, t)|\psi(0)\rangle\langle\psi(0)| \hat{U}^{s \dagger}(\tilde{\phi}, t),
$$

where $\hat{U}^{s}(\phi, t)$ is the stochastic time-evolution operator and we highlight its noise dependence. The density matrix can also be expressed as an integral over the classical coordinates $\xi(t)$ and $\tilde{\xi}(t)$ :

$$
\hat{\rho}(t)=\int d^{2} \xi d^{2} \tilde{\xi} P(\xi) P(\tilde{\xi}) \hat{\rho}^{s}(\xi, \tilde{\xi}),
$$

where $\hat{\rho}^{s}(\xi, \tilde{\xi})=\hat{U}^{s}(\xi)|\psi(0)\rangle\langle\psi(0)| \hat{U}^{s \dagger}(\tilde{\xi})$ and both $P(\xi)$ and $P(\tilde{\xi})$ satisfy the Fokker-Planck equation

$$
\frac{\partial}{\partial t} P(\xi)=\hat{\mathcal{F}} P(\xi)
$$

Here, the differential operator $\hat{\mathcal{F}}$ contains only first- and second-order derivatives with respect to the coordinates $\xi(t)$. Quantum expectation values can be computed within the Fokker-Planck representation by

$$
\langle\hat{\mathcal{O}}(t)\rangle=\int d^{2} \xi d^{2} \tilde{\xi} P(\xi) P(\tilde{\xi}) \operatorname{Tr}\left[\hat{\rho}^{s}(\xi, \tilde{\xi}) \hat{\mathcal{O}}\right] .
$$

Without loss of generality, we may consider initial states that are obtained by time evolution from the spin-down state $|\downarrow\rangle$ [18,19]:

$$
\hat{\rho}^{s}=e^{-\frac{1}{2}\left(\chi+\tilde{\chi}^{*}\right)} \prod_{j}\left|\xi_{j}^{+}\right\rangle\left\langle\tilde{\xi}_{j}^{+}\right|,
$$

where $\left|\xi_{j}^{+}\right\rangle=e^{\xi_{j}^{+} \hat{S}_{j}^{+}}|\downarrow\rangle$ is a spin coherent state and $\chi \equiv$ $\sum_{j} \xi_{j}^{z}$. As we will discuss in Appendixes B and C, Eq. (A5) enables us to make contact with the representation of $\hat{\rho}$ in the phase space literature [21,22]. The Weiss field $m_{j}^{a}$ is interpreted as a drift gauge parameter, which we discuss in Appendix C. To make the connection more explicit we use the parameterization $z_{j}=\ln \left(\xi_{j}^{+}\right)$and $\omega=-\frac{\chi}{2}$, as used in Refs. [22,24]. In this representation

$$
\hat{\rho}^{s}=e^{\omega+\tilde{\omega}^{*}} \prod_{j}\left|z_{j}\right\rangle\left\langle\tilde{z}_{j}\right|,
$$

where

$$
\left|z_{j}\right\rangle=e^{e^{z_{j}} \hat{S}_{j}^{+}}|\downarrow\rangle=|\downarrow\rangle+e^{z_{j}}|\uparrow\rangle
$$

and $z_{j} \in \mathbb{C}$

\section{APPENDIX B: PHASE SPACE REPRESENTATIONS}

In this section we give a brief introduction to the phase space methods developed in Refs. [20-24]. The initial starting point is to consider a general parameterization of a density matrix $\hat{\rho}$ in terms of phase space variables $\lambda$ :

$$
\hat{\rho}=\int d \lambda W(\lambda) \hat{\Lambda}(\lambda),
$$

where $W(\lambda)$ is a quasiprobability distribution and $\hat{\Lambda}(\lambda)$ is an operator kernel. Since $W(\lambda)$ can be negative, it cannot be interpreted as a true probability distribution. However, for bosons [20] and spins [22], $W(\lambda)$ can be made positive by using a generalized kernel built from off-diagonal coherent state projectors: $\hat{\Lambda}\left(\lambda, \lambda^{\prime}\right)=\prod_{j}\left|\lambda_{j}\right\rangle\left\langle\lambda_{j}^{\prime}\right|$, where $\lambda, \lambda^{\prime} \in \mathbb{C}[20,22]$. For example,

$$
\hat{\rho}=\int d^{2} \lambda d^{2} \lambda^{\prime} P\left(\lambda, \lambda^{\prime}\right) \prod_{j} \frac{\left|\lambda_{j}\right\rangle\left\langle\lambda_{j}^{\prime}\right|}{\left\langle\lambda_{j}^{\prime} \mid \lambda_{j}\right\rangle},
$$

where $P\left(\lambda, \lambda^{\prime}\right)$ is positive definite. For bosonic systems, the decomposition (B2) in which the normalization is explicitly enforced is known as the positive- $P$ representation [20]. Analogous representations for spin systems have been considered in Refs. [22,24]. Phase space distributions over coherent states 
are not unique due to the overcompleteness of the basis. This can be exploited by using a more general representation that includes a complex weight $\Omega$. This enlarges the variable space:

$$
\hat{\rho}=\int d^{2} \lambda d^{2} \lambda^{\prime} d^{2} \Omega P\left(\lambda, \lambda^{\prime}, \Omega\right) \Omega \prod_{j}\left|\lambda_{j}\right\rangle\left\langle\lambda_{j}^{\prime}\right| .
$$

With the inclusion of the weight this is referred to as the gauge- $P$ representation [21]. This mirrors Eqs. (A2) and (A6), provided we identify $\Omega=e^{-\frac{1}{2}\left(x+\tilde{\chi}^{*}\right)}$. The integrations over $\lambda$ and $\lambda^{\prime}$ can be further identified as the forwards and backwards time evolutions involving $\xi^{+}$and $\tilde{\xi}^{+}$. Physical observables are calculated according to

$$
\langle\hat{\mathcal{O}}\rangle=\int d^{2} \lambda d^{2} \lambda^{\prime} d^{2} \Omega P\left(\lambda, \lambda^{\prime}, \Omega\right) \Omega \operatorname{Tr}\left(\hat{\Lambda}\left(\lambda, \lambda^{\prime}\right) \hat{\mathcal{O}}\right),
$$

in conformity with Eq. (A4) in the stochastic approach.

Having introduced a formal representation of the density matrix, one may obtain the Fokker-Planck equation by substituting $\hat{\rho}$ into the Liouville equation

$$
i \dot{\hat{\rho}}=[\hat{H}, \hat{\rho}],
$$

where $\hat{H}$ is represented by a differential operator acting on the classical coordinates. Assuming that the Hamiltonian contains no derivative terms higher than second order, one obtains [22]

$$
\frac{\partial}{\partial t} P(\lambda)=\left[V+\sum_{j} \frac{\partial}{\partial \lambda_{j}}\left(-A_{j}+\frac{1}{2} \sum_{l} \frac{\partial}{\partial \lambda_{l}} D_{j l}\right)\right] P(\lambda),
$$

where $\lambda=\left\{\lambda, \lambda^{\prime}\right\}$ and we neglect boundary terms in performing partial integrations. For $V=0$ this is a Fokker-Planck equation, where $A_{j}$ is the drift vector and $D_{j l}$ is the diffusion matrix. The mapping to stochastic Langevin equations can be carried out provided a "noise matrix" $B_{j k}$ exists satisfying $D_{j l}=\sum_{k} B_{j k} B_{l k}$ [20]. The resulting Langevin equations in Ito form are given by [47]

$$
\dot{\lambda}_{j}=A_{j}+\sum_{k} B_{j k} \phi_{k},
$$

where $\phi_{k}$ is Gaussian white noise satisfying

$$
\left\langle\phi_{k}(t) \phi_{k^{\prime}}\left(t^{\prime}\right)\right\rangle=\delta_{k k^{\prime}} \delta\left(t-t^{\prime}\right), \quad\left\langle\phi_{k}(t)\right\rangle=0 .
$$

Observables can be calculated as averages over the noise

$$
\langle\hat{O}\rangle=\langle\Omega \operatorname{Tr}(\hat{\Lambda} \hat{O})\rangle_{\phi},
$$

where $\phi$ includes the forwards and backwards time evolution and $\Omega=1$ is the unweighted case.

\section{APPENDIX C: DRIFT GAUGES}

As discussed in Appendix B, the phase space distribution is not unique. The use of different gauges enables one to move between these representations. The gauges can be introduced by adding a vanishing term to the Liouville equation (B5). Denoting $\Omega=e^{\omega}$, the identity $\left(\frac{\partial}{\partial \omega}-1\right) e^{\omega} \hat{\Lambda}=0$ [21] allows one to add

$$
\int d^{2} \lambda d^{2} \omega P(\lambda, \omega) f(\lambda, \omega)\left(\frac{\partial}{\partial \omega}-1\right) e^{\omega} \hat{\Lambda}=0,
$$

where $f(\lambda, \omega)$ is an arbitrary function. To produce a valid Fokker-Planck equation, without introducing additional noises, $f(\lambda, \omega)$ can be constrained [21]:

$$
f(\lambda, \omega)=V(\lambda)+\frac{1}{2} \sum_{k} g_{k}(\lambda)^{2} \frac{\partial}{\partial \omega}+\sum_{k \alpha} g_{k}(\lambda) B_{\alpha k}(\lambda) \frac{\partial}{\partial \lambda_{\alpha}} .
$$

The first term eliminates $V(\lambda)$ from Eq. (B6), yielding an equation with only first and second derivatives. The functions $g_{k}(\lambda)$ are known as "drift gauges" [21] as they modify the drift terms in Eq. (B7). The resulting Fokker-Planck equation is of the form (B6), but with $A_{\alpha} \rightarrow A_{\alpha}-\sum_{k} g_{k} B_{\alpha k}$ and $D_{\alpha \beta} \rightarrow$ $D_{\alpha \beta}$ left unchanged. One must also introduce additional drift and diffusion terms for the weight variable $\omega$ :

$$
\begin{gathered}
A_{\omega}=V-\frac{1}{2} \sum_{k} g_{k}^{2}, \quad D_{\omega \omega}=\frac{1}{2} \sum_{k} g_{k}^{2}, \\
D_{\alpha \omega}=D_{\omega \alpha}=\sum_{k} g_{k} B_{\alpha k} .
\end{gathered}
$$

The drift of the coherent state parameters $\lambda$ has thus been modified via diffusion and drift in the weight variable $\omega$. The modified Langevin equations are given by

$$
\begin{gathered}
\dot{\lambda}_{\alpha}=A_{\alpha}-\sum_{k} g_{k} B_{\alpha k}+\sum_{k} B_{\alpha k} \phi_{k}, \\
\dot{\omega}=V-\frac{1}{2} \sum_{k} g_{k}^{2}+\sum_{k} g_{k} \phi_{k} .
\end{gathered}
$$

In Appendix D, we use this formalism to link the Weiss field $m_{j}^{a}$ to the drift gauge $g_{k}(\lambda)$.

\section{APPENDIX D: SPIN COHERENT STATES}

In order to make the discussion in Appendixes A, B, and $\mathrm{C}$ more explicit, we introduce spin coherent states following Refs. [22,24]. We consider the spin- $\frac{1}{2}$ state decomposition

$$
|\psi\rangle=\int d^{2} z d^{2} \omega P(z, \omega) e^{\omega} \prod_{j}\left|z_{j}\right\rangle,
$$

where $\omega$ is a complex weight and $\left|z_{j}\right\rangle$ are the un-normalized coherent states defined by (A7). The spin operators are represented by differential operators acting on the coherent state parameters:

$$
\begin{gathered}
\hat{S}_{j}^{z}|z, \omega\rangle=\left(-\frac{1}{2}+\frac{\partial}{\partial z_{j}}\right)|z, \omega\rangle, \\
\hat{S}_{j}^{x}|z, \omega\rangle=\left(\frac{1}{2} e^{z_{j}}-\sinh \left(z_{j}\right) \frac{\partial}{\partial z_{j}}\right)|z, \omega\rangle, \\
\hat{S}_{j}^{y}|z, \omega\rangle=\left(\frac{i}{2} e^{z_{j}}-i \cosh \left(z_{j}\right) \frac{\partial}{\partial z_{j}}\right)|z, \omega\rangle,
\end{gathered}
$$

where $|z, \omega\rangle \equiv e^{\omega} \prod_{j}\left|z_{j}\right\rangle$ are weighted basis states. For a given spin Hamiltonian, we may substitute the decomposition (D1) into the Schrödinger equation, $i \partial_{t}|\psi(t)\rangle=\hat{H}|\psi(t)\rangle$, in order to derive the corresponding Fokker-Planck equation for $P(z, \omega)$. In this representation the analog of $(\mathrm{C} 1)$ is

$$
\int d^{2} z d^{2} \omega P(z, \omega) f(z, \omega)\left(\frac{\partial}{\partial \omega}-1\right)|z, \omega\rangle=0,
$$


where $f(z, \omega)$ is defined by (C2). To begin with we set the gauges $g_{k}(z)$ to zero. However, $V(z)$ must be chosen to remove the zeroth-order terms. Decomposing $\omega$ into on-site contributions $\omega=\sum_{j} \omega_{j}$ yields the SDEs

$$
\begin{aligned}
& -i \dot{z}_{j}=\Phi_{j}^{z}-\Gamma \sinh \left(z_{j}\right)-\frac{1}{2} \sum_{l} J_{j l}, \\
& -i \dot{\omega}_{j}=-i V_{j}(z) \equiv \frac{\Gamma}{2} e^{z_{j}}+\sum_{l} \frac{1}{8} J_{j l},
\end{aligned}
$$

where $\Phi_{j}^{z}=-\sum_{k} i B_{j k} \phi_{k}$ is given in terms of the independent white noises $\phi_{k}$ and we have set $V=\sum_{j} V_{j}$. The "noise matrix" $B_{j k}$ is defined via the diffusion matrix $D_{j l}=i J_{j l}=$ $\sum_{k} B_{j k} B_{l k}$. We can make contact with the SDEs (4a) and (4b) discussed in the main text, including the Weiss field $m_{j}^{z}$, by introducing the drift gauge

$$
g_{k}=-\sum_{j}\left(\frac{1}{2}+m_{j}^{z}\right) B_{j k}
$$

The SDEs (D6) and (D7) become

$$
\begin{gathered}
-i \dot{z}_{j}=\Phi_{j}^{z}-\Gamma \sinh \left(z_{j}\right), \\
-i \dot{\omega}_{j}=\frac{\Gamma}{2} e^{z_{j}}-\frac{1}{2} \Phi_{j}^{z}+i m_{j}^{z} \sum_{k} B_{j k} \phi_{k},
\end{gathered}
$$

where now $\Phi_{j}^{z}=\sum_{l} J_{j l} m_{l}^{z}-\sum_{k} i B_{j k} \phi_{k}$. The variables used here can be related to those in the SDEs (4a) and (4b) via the identification $z_{j}=\ln \xi_{j}^{+}, \omega_{j}=-\frac{\xi_{j}^{z}}{2}$, and $B_{j k}=\frac{1}{\sqrt{i}} O_{j k}^{z z}$. In writing (D10) we have neglected the contribution $\frac{1}{2} \sum_{k} g_{k}^{2}$ since it results in a deterministic phase for $\left|\psi^{s}(t)\right\rangle$ which is identical for all trajectories. The additional noise term in (D10) appears via the noise action (7), rather than the SDE (4b) for $\xi_{j}^{z}$; the two are equivalent since $\omega$ enters via $e^{\omega}$ in (D1).

\section{APPENDIX E: WEISS-FIELD CALCULATION}

To calculate the time-evolving Weiss field for the simulations of the Ising model presented in the main text we use the following procedure. In the first step we take $\mathcal{N}$ samples of the SDEs (9a) and (9b) with $m_{j}^{z}(t)=0$, where we use a two-patch approach to avoid coordinate singularities in (9a) [18]. In the second step, we use these trajectories to calculate $m_{j}^{z}(t)$ via (14). For translationally invariant systems we consider a single Weiss field obtained from the spatial average $m^{z}(t)=\frac{1}{N} \sum_{j} m_{j}^{z}(t)$. We then use this as the local Weiss field in the next simulation. We repeat steps 1 and 2 until $m^{z}(t)$ converges to a sufficient level of accuracy over the timescales of interest or until it decays to zero. The resulting Weiss field can now be used in simulations to obtain quantum observables. As discussed above, throughout this work we take the spatial average over the entire system to estimate $m^{z}(t)$. However, as noted in the main text, it can, in principle, be calculated from a small subsystem which captures the local interactions between the spins. To see this, it is convenient to introduce a variant of the Hubbard-Stratonovich transformation which places decoupling fields on the bonds between the spins, rather than on the sites of the lattice. We consider again the

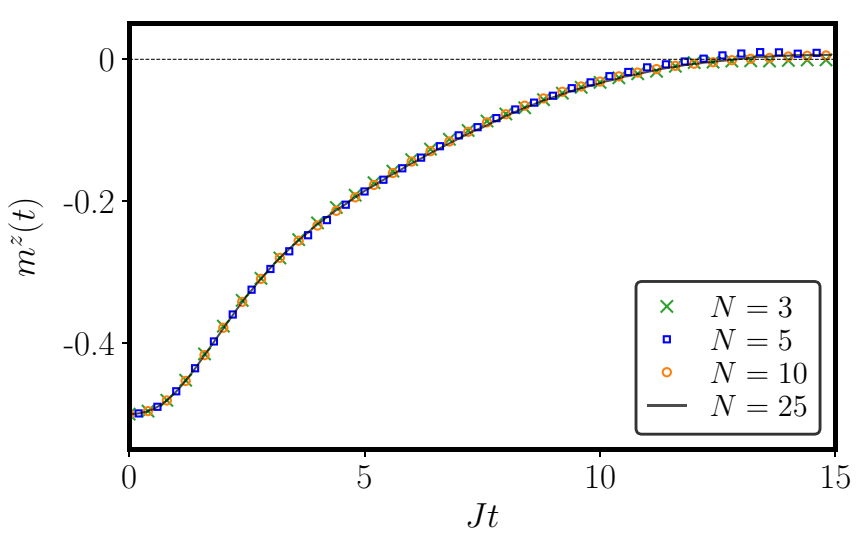

FIG. 6. Time-dependent Weiss field $m^{z}(t)$ following a quantum quench in the 1D quantum Ising model from the fully polarized state $|\Downarrow\rangle$ to $\Gamma / J=0.3$ for different system sizes. The simulations are carried out using site noise, with four iterations of $\mathcal{N}=5000$ stochastic samples and $d t=0.1$. Only the final iteration is shown.

generic quadratic spin Hamiltonian (1). The interactions in the time-evolution operator can be decoupled by performing an integral transformation over auxiliary fields $\eta_{i j}^{a b} \in \mathbb{C}$, which correspond to the interactions between spins:

$$
\hat{U}\left(t_{f}, t_{i}\right)=\mathbb{T} \int \mathcal{D} \eta \mathcal{D} \eta^{*} e^{-S\left[\eta, \eta^{*}\right]+i \int d t \sum_{j a} \Phi_{j}^{a} \hat{S}_{j}^{a}} .
$$

Here, $\mathcal{D} \eta \mathcal{D} \eta^{*}=\prod^{\prime} \mathcal{D} \eta_{i j}^{a b} \mathcal{D} \eta_{i j}^{a b *}$, where the prime indicates that the product is over the bonds linking the spins. We label every spin in the array, in arbitrary dimension, with numbers 1 to $N$, so that $\eta_{i j}^{a b}$ is associated with the bond between sites $i$ and $j$. The effective magnetic field $\Phi_{i}^{a}$ is given by

$$
\Phi_{i}^{a}(t)=\frac{1}{\sqrt{i}} \sum_{\substack{b j \\(j<i)}}^{\prime} \eta_{i j}^{a b}+\frac{1}{\sqrt{i}} \sum_{\substack{b j \\(j>i)}}^{\prime} \eta_{j i}^{b a *}+h_{i}^{a},
$$

where the prime indicates that the summation is restricted to bonds. The path integral weight is given by

$$
S\left[\eta, \eta^{*}\right]=\int_{t_{1}}^{t_{2}} d t \sum_{i j a b}^{\prime} \frac{1}{J_{i j}^{a b}} \eta_{i j}^{a b}(t) \eta_{i j}^{a b *}(t) .
$$

The Gaussian "bond noises" satisfy $\left\langle\eta_{i j}^{a b}(t) \eta_{k l}^{c d *}\left(t^{\prime}\right)\right\rangle=J_{i j}^{a b} \delta_{i k}$ $\delta_{j l} \delta^{a c} \delta^{b d} \delta\left(t-t^{\prime}\right)$, with $\left\langle\eta_{i j}^{a b}(t)\right\rangle=0$ and $\left\langle\eta_{i j}^{a c}(t) \eta_{k l}^{b d}\left(t^{\prime}\right)\right\rangle=0$. Since the number of bond noises scales with the coordination number and each complex noise is the sum of two real noises, they are computationally more intensive to draw numerically than site-based noises. However, bond noises can offer some advantages for Weiss field estimation. For example, in the case of nearest-neighbor interactions, the spins that are not nearest neighbors will evolve independently. In addition, since every spin experiences fluctuations of the same strength they will have identical mean-field dynamics if the initial state is translationally invariant. As a result, in this case it is possible to calculate the time-evolving Weiss field from a single spin, coupled to its nearest neighbors. Adding additional spins does not change the stochastic evolution of the selected spin.

A similar approach to Weiss field estimation can be taken using site noise, but it requires additional justification. Recall 
that the stochastic magnetic field in direction $a$ experienced by each spin is given by $\frac{1}{\sqrt{i}} \varphi_{j}^{a}$, where $\varphi_{j}^{a}=\sum_{k b} O_{j k}^{a b} \phi_{k}^{b}$. In general, the matrix $O_{j k}^{a b}$ ensures that the spins do not evolve independently since they experience common noise fields $\phi_{k}^{b}$. However, only the strength of the noise is relevant for Weiss field estimation as (15) is an average for a single spin; the correlations between spins on individual stochastic trajectories are not required. In particular, if $\sum_{k b}\left|O_{j k}^{a b}\right|^{2}$ is translationally invariant (i.e., independent of $j$ ) and we consider translationally invariant initial states, the Weiss field can be estimated from $N^{-1} \sum_{j} m_{j}^{a}(t)$. This is true for all the simulations considered in this work.

As in the case of bond noise, it is possible to estimate the Weiss field from an appropriate subsystem that reflects the local interactions, provided that $\sum_{k b}\left|O_{j k}^{a b}\right|^{2}$ is approxi- mately independent of the system size. For nearest-neighbor interactions, we find that it indeed exhibits only very weak $N$ dependence. For example, for the $N=101$ site system simulated in Fig. 5(a), $\sqrt{\sum_{k}\left|O_{j k}^{z z}\right|^{2}} \approx 1.128$, while for an $N=3$ spin system $\sqrt{\sum_{k}\left|O_{j k}^{z z}\right|^{2}} \approx 1.155$, corresponding to a $2 \%$ difference. In Fig. 6 we demonstrate the similarity in the extracted Weiss field for a range of system sizes following a quantum quench in the 1D quantum Ising model. For the nearest-neighbor $2 \mathrm{D}$ simulation of an $11 \times 11$ lattice in Fig. 5(b) the difference in $\sqrt{\sum_{k}\left|O_{j k}^{z z}\right|^{2}}$ compared with a $3 \times 3$ system is approximately $4.3 \%$. Given the effectiveness of a well-chosen static Weiss field, this difference is not expected to be significant. It should therefore be possible to estimate the Weiss field by using a smaller system size.
[1] M. Rigol, V. Dunjko, V. Yurovsky, and M. Olshanii, Phys. Rev. Lett. 98, 050405 (2007).

[2] M. Rigol, V. Dunjko, and M. Olshanii, Nature (London) 452, 854 (2008).

[3] M. Heyl, A. Polkovnikov, and S. Kehrein, Phys. Rev. Lett. 110, 135704 (2013).

[4] M. Heyl, Rep. Prog. Phys. 81, 054001 (2018).

[5] P. Jurcevic, H. Shen, P. Hauke, C. Maier, T. Brydges, C. Hempel, B. P. Lanyon, M. Heyl, R. Blatt, and C. F. Roos, Phys. Rev. Lett. 119, 080501 (2017).

[6] S. R. White and A. E. Feiguin, Phys. Rev. Lett. 93, 076401 (2004).

[7] G. Vidal, Phys. Rev. Lett. 93, 040502 (2004).

[8] J. Haegeman, J. I. Cirac, T. J. Osborne, I. Pižorn, H. Verschelde, and F. Verstraete, Phys. Rev. Lett. 107, 070601 (2011).

[9] S. Paeckel, T. Köhler, A. Swoboda, S. R. Manmana, U. Schollwöck, and C. Hubig, Ann. Phys. (NY) 411, 167998 (2019).

[10] P. Czarnik, J. Dziarmaga, and P. Corboz, Phys. Rev. B 99, 035115 (2019).

[11] C. Hubig, A. Bohrdt, M. Knap, F. Grusdt, and J. I. Cirac, SciPost Phys. 8, 021 (2020).

[12] M. P. Zaletel and F. Pollmann, Phys. Rev. Lett. 124, 037201 (2020).

[13] P. M. Hogan and J. T. Chalker, J. Phys. A 37, 11751 (2004).

[14] V. Galitski, Phys. Rev. A 84, 012118 (2011).

[15] M. Ringel and V. Gritsev, Phys. Rev. A 88, 062105 (2013).

[16] S. De Nicola, B. Doyon, and M. J. Bhaseen, J. Phys. A 52, 05LT02 (2019).

[17] S. De Nicola, B. Doyon, and M. J. Bhaseen, J. Stat. Mech. (2020) 013106.

[18] S. E. Begg, A. G. Green, and M. J. Bhaseen, J. Phys. A 53, 50LT02 (2020).

[19] S. De Nicola, J. Stat. Mech. (2021) 013101.

[20] P. D. Drummond and C. W. Gardiner, J. Phys. A 13, 2353 (1980).

[21] P. Deuar and P. D. Drummond, Phys. Rev. A 66, 033812 (2002).

[22] D. W. Barry and P. D. Drummond, Phys. Rev. A 78, 052108 (2008).

[23] R. Ng and E. S. Sørensen, J. Phys. A 44, 065305 (2011).

[24] R. Ng, E. S. Sørensen, and P. Deuar, Phys. Rev. B 88, 144304 (2013).
[25] S. Mandt, D. Sadri, A. A. Houck, and H. E. Türeci, New J. Phys. 17, 053018 (2015).

[26] S. Wüster, J. F. Corney, J. M. Rost, and P. Deuar, Phys. Rev. E 96, 013309 (2017).

[27] P. Deuar, A. Ferrier, M. Matuszewski, G. Orso, and M. H. Szymańska, PRX Quantum 2, 010319 (2021).

[28] M. J. Steel, M. K. Olsen, L. I. Plimak, P. D. Drummond, S. M. Tan, M. J. Collett, D. F. Walls, and R. Graham, Phys. Rev. A 58, 4824 (1998).

[29] A. Polkovnikov, K. Sengupta, A. Silva, and M. Vengalattore, Rev. Mod. Phys. 83, 863 (2011).

[30] J. Schachenmayer, A. Pikovski, and A. M. Rey, New J. Phys. 17, 065009 (2015).

[31] J. Schachenmayer, A. Pikovski, and A. M. Rey, Phys. Rev. X 5, 011022 (2015).

[32] R. Khasseh, A. Russomanno, M. Schmitt, M. Heyl, and R. Fazio, Phys. Rev. B 102, 014303 (2020).

[33] J. Huber, P. Kirton, and P. Rabl, Phys. Rev. A 102, 012219 (2020).

[34] W. Verstraelen and M. Wouters, Appl. Sci 8, 1427 (2018).

[35] W. Verstraelen, R. Rota, V. Savona, and M. Wouters, Phys. Rev. Res. 2, 022037(R) (2020).

[36] P. Deuar and P. D. Drummond, Comput. Phys. Commun. 142, 442 (2001).

[37] P. D. Drummond and P. Deuar, J. Opt. B 5, S281 (2003).

[38] P. D. Drummond, P. Deuar, and K. V. Kheruntsyan, Phys. Rev. Lett. 92, 040405 (2004).

[39] W. Rümelin, SIAM J. Numer. Anal. 19, 604 (1982).

[40] P. E. Klöden and E. Platen, Numerical Solution of Stochastic Differential Equations (Springer, Berlin, 1992).

[41] S. De Nicola, Ph.D. thesis, King's College London, 2018.

[42] M. P. Zaletel, R. S. K. Mong, C. Karrasch, J. E. Moore, and F. Pollmann, Phys. Rev. B 91, 165112 (2015).

[43] R. A. Horn and C. R. Johnson, Matrix Analysis (Cambridge University Press, Cambridge, 2012).

[44] P. Weinberg and M. Bukov, SciPost Phys. 7, 020 (2019).

[45] M. Fishman, S. R. White, and E. M. Stoudenmire, arXiv:2007.14822.

[46] S. De Nicola, arXiv:2103.16468.

[47] H. Risken, The Fokker-Planck Equation (Springer, Berlin, 1984). 